\title{
A Dynamic Clustering Algorithm for MANETs by modifying Weighted Clustering Algorithm with Mobility Prediction
}

\author{
S.Muthuramalingam, R.RajaRam, Kothai Pethaperumal and V.Karthiga Devi
}

\begin{abstract}
The multi hop packet radio networks also named mobile ad-hoc networks (MANETs) have a dynamic topology due to the mobility of their nodes. A notable amount of energy is utilized every time a signal is sent and received by a mobile node. Many such signals and power are wasted to update the positional information of the nodes in a wireless scenario. Further bandwidth is also wasted by sending control signals rather than using it effectively for data communication. To minimize this utilization, we propose a modified algorithm that uses Weighted Clustering Algorithm (WCA) for cluster formation and Mobility Prediction for cluster maintenance. Clustering is an effective technique for node management in a MANET. Cluster formation involves election of a mobile node as Cluster head and it controls the other nodes in the newly formed cluster. The connections between nodes and the cluster head changes rapidly in a mobile ad-hoc network. Thus cluster maintenance is also essential. Prediction of mobility based cluster maintenance involves the process of finding out the next position that a mobile node might take based on the previous locations it visited. In this paper we propose to reduce the overhead in communication by predicting mobility of node using linear auto regression and cluster formation.
\end{abstract}

Index Terms - Ad-hoc, Clustering, Cluster-Head, Mobility Prediction.

\section{INTRODUCTION}

The rapid advancement in mobile computing platform and wireless communication technology lead us to develop a method to elect cluster-heads and form clusters [5] in wireless mobile ad-hoc networks [9]. These networks where fixed infra structure does not exist permit the interconnectivity between work-groups moving in urban and rural areas. They can also help in Collaborative operations, for example, distributed scientific research or rescue.

A wireless ad hoc network [8] is a decentralized wireless network the network is ad hoc because each node is willing to forward data for other nodes, and so the determination of which nodes forward data is made dynamically based on the network connectivity.

Multi-cluster [5], multi-hop wireless network should be able to dynamically adopt itself. Some nodes, known as cluster-heads, are responsible for formation of clusters each consisting of number of nodes (analogous to cells in a cellular network) and maintenance of topology of network. The set of cluster-heads is also called Dominant set[9]. A cluster-head is responsible for resource allocation to all nodes belonging to its cluster and monitors communication within a cluster. In a cluster [5], objects are mutually closer to each other than to objects in other clusters. The Cluster structure need to be maintained as the new mobile nodes may enter the network and the existing nodes may move out or lose their battery power [1]. It occurs in the case of both Cluster-Heads and Member Nodes.

Prediction of the geographical position of the Mobile Node is called mobility prediction. The Linear Auto Regression is used, among the many techniques available for predicting. The past positions or the history is used in predicting the future positions. Based on this value Clustering is performed. When it is compared to the Original Position the resulting Cluster Formed are the same. Thus Signals sent from the member nodes to the Cluster-Head regarding the Current Position can be minimized. This will reduce the Consumption of Power [1], Wastage of Bandwidth for signals other than Data, and ultimately Increase the Stability of the Cluster [11][18].

\section{RELATED WORK}

Recently, a number of clustering algorithms have been proposed and based on some of criteria to choose cluster-head such as speed and direction, mobility [2, 3], energy, position, and the number of neighbors of a given node. These works present advantages but some drawbacks as a high computational overhead for both clustering algorithm execution and update operations. We will give each of them a brief description as follows:

The Highest-Degree Algorithm, also known as connectivity-based algorithm [12, 19]. This algorithm is based on the degree of nodes assumed to be the number of neighbors of a given node. Whenever the election procedure is needed, nodes broadcast their Identifier (ID) which is assumed to be unique in the same network. According to the number of received IDs every node computes its degree and the one having the maximum degree becomes cluster-head. Major drawbacks of this algorithm are the degree of a node changes very frequently, the $\mathrm{CHs}$ are not likely to play their role as cluster-heads for very long. In addition, as the number of ordinary nodes in a cluster is increased, the throughput drops and system performance degrades. All these drawbacks occur because this approach does not have any restriction on the upper bound on the number of nodes in a cluster.

The Lowest-Identifier (LID) also known as identifier-based clustering algorithm, [13] chooses the node with the lowest ID as a cluster-head, the system performance 
is better than Highest-Degree in terms of throughput. Major drawbacks of this algorithm are its bias towards nodes with smaller ids which may lead to the battery drainage of certain nodes, and it does not attempt to balance the load uniformly across all the nodes. The Distributed Clustering Algorithm (DCA) [10][19] and Distributed Mobility Adaptive Clustering Algorithm (DMAC) [15][19]. In this approach, each node is assigned weights [4] (a real number above zero) based on its suitability of being a cluster-head. A node is chosen to be a cluster-head if its weight is higher than any of its neighbor's weight [17]; otherwise, it joins a neighboring cluster-head. The smaller node id [7] is chosen in case of a tie. The DCA makes an assumption that the network topology does not change during the execution of the algorithm. To verify the performance of the system, the nodes were assigned weights which varied linearly with their speeds [2] [3] but with negative slope. Results proved that the number of updates required is smaller than the Highest-Degree and Lowest-ID heuristics. Since node weights [4] [7] were varied in each simulation cycle, computing the cluster-heads becomes very expensive and there are no optimizations on the system parameters such as throughput and power control [6].

The Weighted Clustering Algorithm (WCA) [4] [8] [14] obtains 1-hop clusters with one cluster-head. The election of the cluster-head is based on the weight of each node [17]. It takes four factors into consideration and makes the selection of cluster-head and maintenance of cluster more reasonable. The four factors are node degree (number of neighbors), distance summation to all its neighboring nodes, mobility [2] [3] and remaining battery power [1]. Although WCA has proved better performance than all the previous algorithms, it lacks a drawback in knowing the weights of all the nodes before starting the clustering process and in draining the $\mathrm{CHs}$ rapidly. As a result, the overhead induced by WCA is very high.

\section{PROPOSED WORK}

\section{A. Assumption}

The following assumptions are made before clustering

1) The network topology is static during the execution of the clustering algorithm.

2) Each mobile node joins exactly one cluster-head.

3) The optimal number of nodes in the cluster $\alpha$ is assumed to be 8 .

4) The co-efficient used in Weight calculations are assumed the following values, w1 $=0.7$, w2 $=0.2$, $\mathrm{w} 3=0.05, \mathrm{w} 4=0.05$. The sum of these co-efficient is 1 . This is actually used to normalize the factors such as spreading degree, distance with its neighbors, mobility of the node, and power consumed used in the calculation of weight of a node. The factors spreading degree and distance with its neighbors are given more importance and assumed higher co-efficient values 0.7 and 0.2 respectively.

\section{B. Formation of Cluster}

Initially, each node broadcasts a beacon message to notify its presence to the neighbors. A beacon message contains the state of the node. Each node builds its neighbor list based on the beacon messages received. The cluster-heads

Election is based on the weight values [4] [7] of the nodes and the node having the lowest weight is chosen as $\mathrm{CH}$ [17]. Each node computes its weight value based on the following algorithm:

Step 1: The coefficients used in weight calculation are assumed the following values $\mathrm{w} 1=0.7, \mathrm{w} 2=0.2, \mathrm{w} 3=0.05$, $\mathrm{w} 4=0.05$; Based on the assumption in section 3.1 .

Step 2: Compute the difference between the optimal cluster's size ' $\alpha$ ' and the real number of neighbors ' $R(V)$ ' as spreading degree,

$\Delta \mathrm{sp}=1-(|\alpha-\mathrm{R}(\mathrm{V})| / \alpha)$

Step 3: For every node the sum of the distances, Dv, with all its neighbors is calculated.

$\mathrm{Dv}=\sum \operatorname{dist}\left(\mathrm{v}, \mathrm{v}^{\prime}\right)$ where $\mathrm{v}^{\prime} \mathrm{eN}(\mathrm{v})$

Step 4: Calculate the average speed for every node until the current time $\mathrm{T}$. This gives the measure of the mobility $\mathrm{Mv}$ [2] [3] based on the X co-ordinate and Y co-ordinate ie., position of the node $\mathrm{v}$ at all previous time instance $\mathrm{t}$.

Step 5: Determine how much battery power has been consumed as $\mathrm{Pv}$. This is assumed to be more for a Cluster-Head [5] when compared to an ordinary node. Because Cluster-Head has taken care of all the members of the cluster by continuosly sending the signal.

Step 6: The weight $\mathrm{Wv}$ for each node is calculated based on

$$
W v=(w 1 \times \Delta s p)+(w 2 \times D v)+(w 3 \times M v)+(w 4 \times P v)
$$

Where $\Delta \mathrm{sp}$ is the spreading degree, Dy is the distance with its neighbors, My is the mobility of the node, and power consumed is represented by, $\mathrm{Pv}$

Step 7: The node with the smallest $\mathrm{Wv}$ is elected as a cluster-head. All the neighbors of the chosen cluster-head are no more allowed to participate in the election procedure.

Step 8: All the above steps are repeated for remaining nodes which is not yet elected as a cluster-head or assigned to a cluster. 
MODIFIED WEIGHTED CLUSTERING ALGORITHM FOR A PARTICULAR NODE NAMED ' $\mathrm{V}$ '

\section{ACTION 1:}

Broadcast a beacon signal to all its neighbor nodes in the transmission range;

Process the beacon signals received from the neighbor nodes in the network and form the connection matrix, A;

\section{ACTION 2:}

Calculate Weight of node $\mathrm{V}$, as $\mathrm{W}_{\mathrm{v}}$;

\section{ACTION 3:}

Broadcast weight value Wv to all its neighbor nodes;

Process the signals received from the neighbor nodes in the network and identify the weights of the neighbors;

\section{ACTION 4:}

Find the node with minimum weight in the neighborhood;

If ( $\mathrm{W}_{\mathrm{v}}$ is the least weight)

Declare itself as the Cluster-head; Else

Send request to join the Cluster formed by the neighbor with least weight;

An example of four clusters with four cluster-heads and different number of member nodes is shown below.

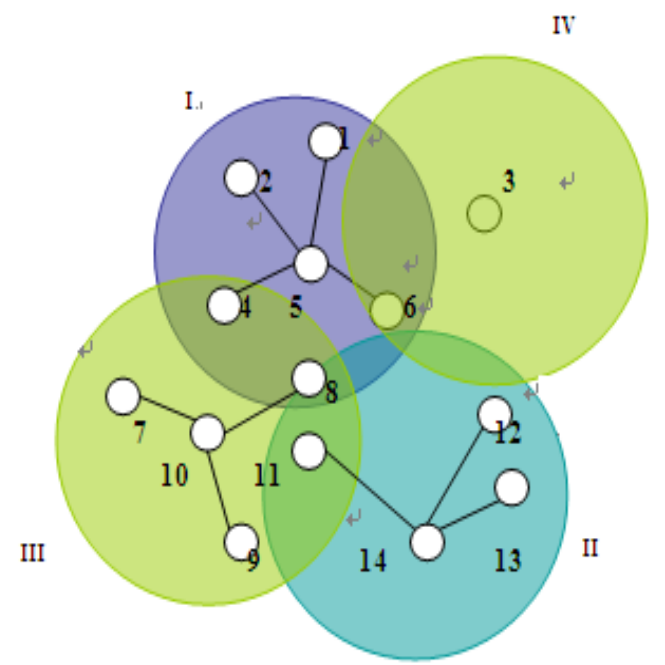

The cluster I has the Node 1 , Node 2 , Node 4, Node 5, and Node 6 . These have the head as Node 5 . The clusters do not share the Node with any other cluster.

The composition of the Cluster II is Node 13, Node 14, Node 11 and Node 12. The Node 14 is the Cluster-Head elected in this scenario.

Similarly the composition of the Cluster III is Node 10, Node 7, Node 8 and Node 9. The Cluster-head is Node 10.

There is a secluded Node 3 which forms a cluster of its own as it does not have any other connection for weight calculation.

The dominant set has the Node 3 , Node 10, Node 14, Node 5.

\section{Cluster Maintenance}

The second phase is the clustering maintenance. There are defined two distinct types of operations for cluster maintenance: the battery power [1] threshold property and the node movement to the outside of its cluster boundary.

1) Node Movements

The node movements can be in the form of node joining or node leaving a cluster. These operations will have only local effects on the clustered topology if the moving node is a CM node. If the leaving node is $\mathrm{CH}$ node, the cluster reorganization has to be performed for the nodes in the cluster by evoking the clustering algorithm.

The Concept of Linear Auto Regression is, given a time series of data, the autoregressive (AR) models is a tool for understanding and predicting future values in this series. It is used in statistics and signal processing. For $i=1 \ldots \mathrm{P}$

$$
\mathrm{X} \tau=\mathrm{X} \tau-i+\left(\sum((\mathrm{X} \tau-i)-(\mathrm{X} \tau-j)) \div N\right)
$$

Where $X \tau$ is the predicted value at time $\tau$ based on the average rate of change of the previous values. $\mathrm{N}$ is the total number of differences calculated. The value of $i$ and $j$ are 1 and 2 respectively.

Using the regression technique a time series data about the previous position of the node are analyzed and the next value in the series is predicted. Using this predicted value [4] [8] [14] the cluster calculation is done.

It is found that the cluster formed with predicted values and actual values are the same and thus the power of the mobile node [6] can be saved if we use prediction calculation and avoid the beacon signals between mobile node and its Cluster-Head to get the geographical position of the node. Bandwidth that must be utilized for data transfer is also saved in this case. A stable cluster topology is thus obtained.

\section{ALGORITHM FOR NEWLY ARRIVING NODE 'U'}

ACTION 1:

Broadcast a beacon signal to all its neighbor nodes in the transmission range;

Process the signals received from the neighbor nodes in the network and form the connection matrix, A;

\section{ACTION 2:}

Calculate the Degree R (U) using A, Spreading degree, $\Delta s p$, Sum of the distances, $D_{u}$, with all its neighbors, Average speed, $\mathrm{M}_{\mathrm{u}}$, Amount of battery power that has been consumed, $\mathrm{P}_{\mathrm{u}}$, and Weight of node $\mathrm{U}$ as $\mathrm{W}_{\mathrm{u}}$;

\section{ACTION 3:}

If(A Cluster head already exist in the neighborhood) Else Send request to join the Cluster;

Form a new cluster and declare itself as the cluster-head;

\section{2) Battery Power Threshold}

The battery power of the nodes participating in the Clustering changes continuously. The Cluster-Heads Power 
[1] decreases more rapidly when compared to the Cluster Members. When the Cluster-Heads Battery Power falls below a threshold then the node is no longer able to perform its activates and a New Head from the members available need to be chosen.

CLUSTER-HEAD
ACTION:
Verify the threshold on the Cluster -Head's Battery power;
If (Battery power < Threshold)
Cluster-Head sends a
LIFE_DOWN message to all its
Neighbors;
All the Member nodes participate
in the Re-Election Procedure
using Modified Weighted
Clustering Algorithm and the
Node with least weight is
selected as the New Cluster-
Head;
Else
Re-election is not needed;

\section{Performance Evaluation}

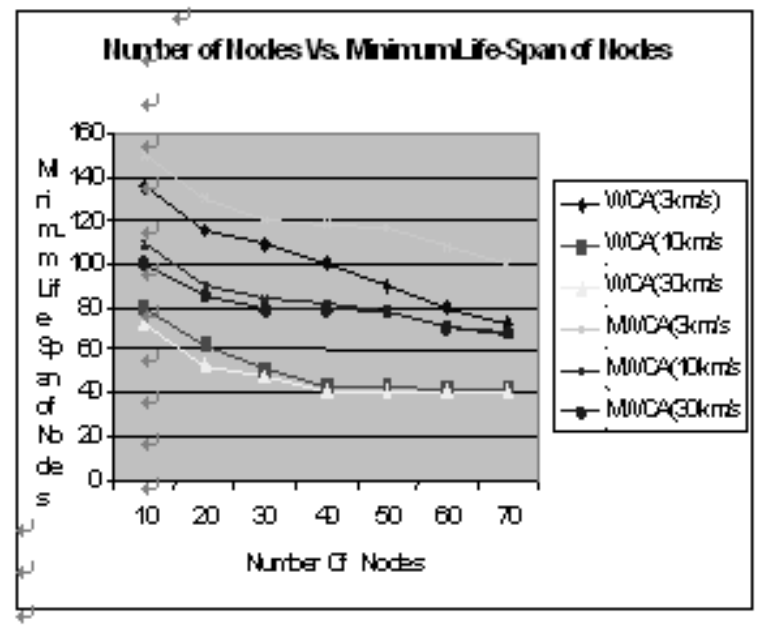

Figure 4.1

The Graph in figure 4.1 specifies the advantage of using Modified Weighted Clustering Algorithm and Mobility Prediction over the Static Weighted Clustering Algorithm. It was computed for varying number of mobile nodes 10,20 , $30,40,50,60$, and 70 in a NS2 simulation environment. Based on the Calculation of Life span of the nodes the Modified WCA with Mobility Prediction is proved to perform better than WCA. The Life span of the Node is inversely proportional to the Speed with which the node travels. The Life span of the Node is higher in all the cases of $3 \mathrm{~km} / \mathrm{s}, 10 \mathrm{~km} / \mathrm{s}$ and $30 \mathrm{~km} / \mathrm{s}$ for the nodes clustered using Modified WCA than WCA with corresponding values. Vast difference is found in the case of $\operatorname{MWCA}(3 \mathrm{~km} / \mathrm{s})$ as it is proved to be much better than WCA. Using NS2 simulation environment the behavior of different node population is observed and minimal life span is found to be highest in modified WCA. Thus the lower limits of minimum life span increases. Basically for a node moving with lesser speed the
Life-span is longer. This feature is more enhanced in the case of our new Algorithm.

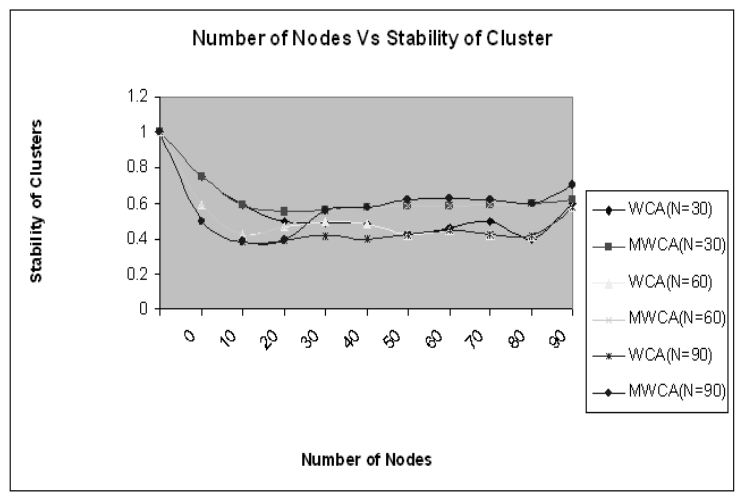

Figure 4.2

In figure 4.2 Stability of the Cluster formed using WCA and Modified WCA with other algorithms is compared [11, 18]. Computation for varying number of mobile nodes 10,20 , $30,40,50,60$, and 70 in a NS2 simulation environment. When the number of nodes is zero all the clusters are highly stable. But when the number of nodes increases the Stability gradually decreases and stabilizes in the range of 40 to 80 nodes. This stable value for the Modified WCA is higher in the range of 0.7 than the WCA which is in the range of 0.5 . Stability is a essential criteria for efficient clustering and using NS2 simulation the Modified weighted clustering algorithm(MWCA) is proved to have higher stability value than the Weighted clustering algorithm(WCA).

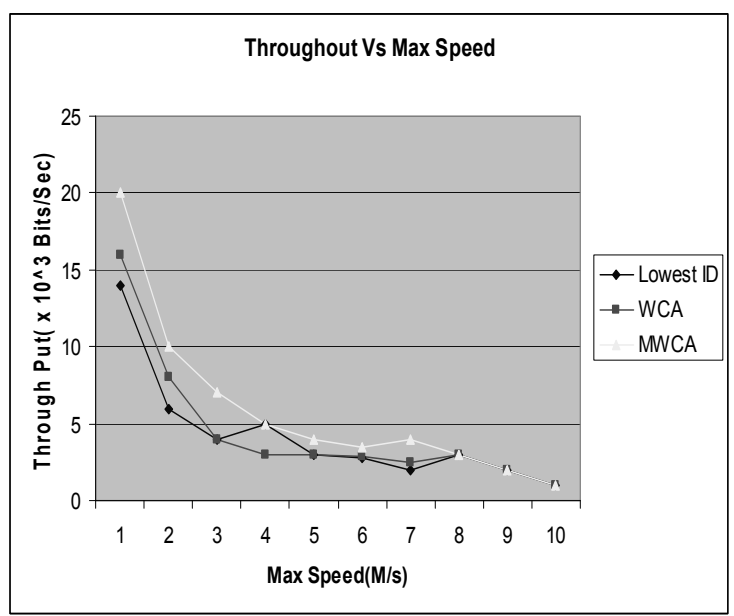

Figure 4.3

NS2 simulation for an optimal 50 nodes is carried out to observer the variation in throughput and Maximum speed of movement which are inversely related. Throughput is the amount of useful information transmitted between the Nodes. Basically the Throughput decreases when the Speed with which the node travels increases. It is represented graphically in figure 4.3. At every level of Speed the Three types of Clustering Lowest ID, WCA and Modified Weighted Clustering Algorithm are compared. At the speeds from $1 \mathrm{~m} / \mathrm{s}$ to $7 \mathrm{~m} / \mathrm{s}$ the Modified Weighted Clustering Algorithm has better throughput than any other Clustering algorithm. But when the Speed increases to High values like $8 \mathrm{~m} / \mathrm{s}, 9 \mathrm{~m} / \mathrm{s}$, 
and $10 \mathrm{~m} / \mathrm{s}$ the throughput of all the algorithm is drastically low. In such cases the Power utilization of the nodes are high.

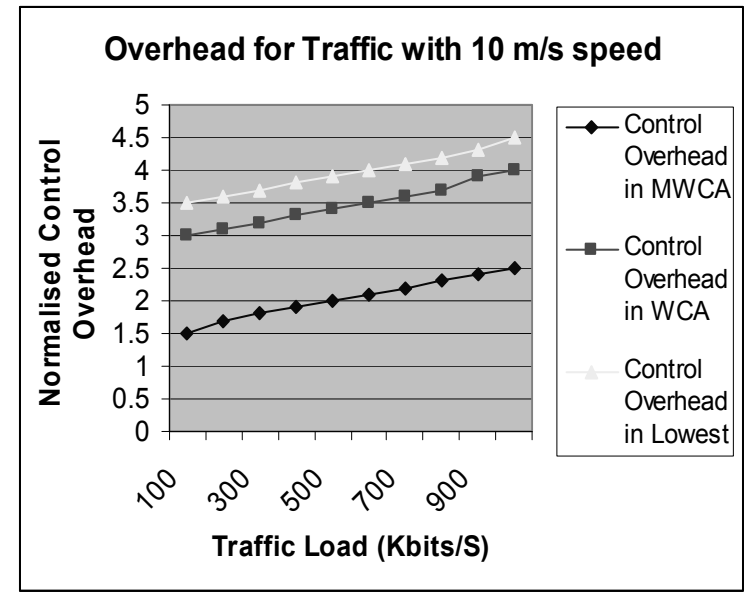

Figure 4.4

Lower the traffic generation rate higher is the availability of network resources as shown in figure 4.4. The number of sources is 100 with varying traffic generation rated and speed used for this analysis. NS2 simulation for 100 nodes is executed and the traffic load and the Normalized Control overhead values are tabulated. When the traffic load increases the Control required and overhead involved also increases. Thus the overall normalized control overhead increases. In case of Modified Weighted Clustering algorithm(MWCA) the overhead is minimum because cluster is maintained using predicted mobility with high accuracy. As the mobile nodes position is predicted the overhead involved in maintaining the signal based position discovery is avoided. Traffic load measured in Kbits/S is varied from $100 \mathrm{Kbits} / \mathrm{s}$ to $900 \mathrm{Kbits} / \mathrm{s}$ and the corresponding Control overhead is measured in a scale of 5 and it is unit less.

Effective utilization of power, Minimum wastage of Bandwidth, More Stable Clusters help in improving the overall Life-span of a node and thus the lower limits of minimum life span increases. Basically for a node moving with lesser speed the Life-span is longer. This feature is more enhanced in the case of our new Algorithm.

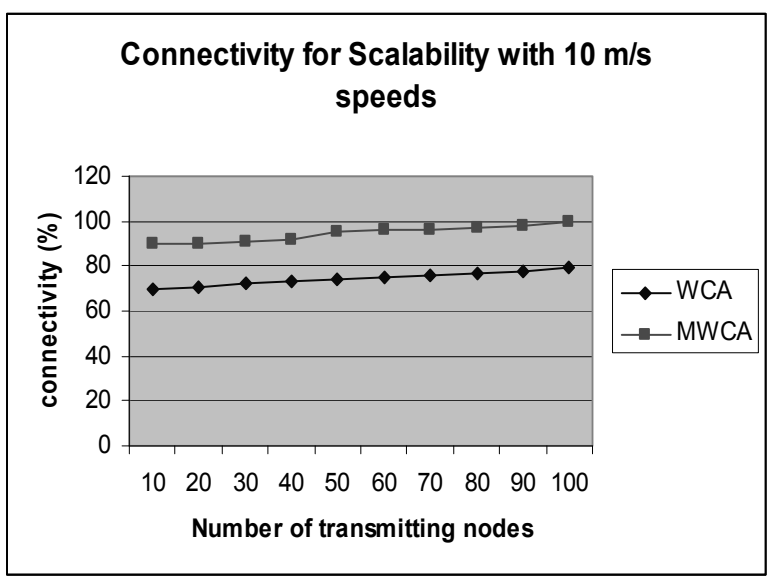

Figure 4.5

The Scalability analysis is done in figure 4.5 by varying the number of source nodes in the network to create congestion. The Source variations range from 10 to 100 mobile node pairs. The speed of the source nodes is varying at $10 \mathrm{~m} / \mathrm{s}$. The connectivity maintenance gives the number of source nodes connected. The Connectivity of nodes gives path for data packets to be forwarded without loss and comparison results are shown in the above graph.NS2 simulation with varying node count ranging from 10 to 100 is used to study the percentage of connectivity in wireless environment. Maximum possible connectivity is $100 \%$ where each node is connected with every other node.

Modified Weighted Clustering Algorithm(MWCA) shows $100 \%$ connectivity as the number of transmitting nodes increases.

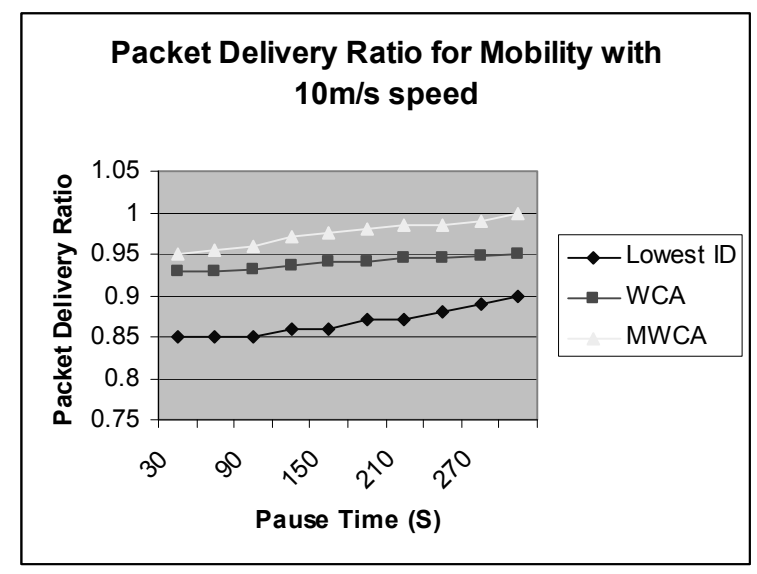

Figure 4.6

Mobility analysis in NS2 simulated environment with 100 nodes is given in figure 4.6 with different Pause time values which influence the node mobility in the random waypoint model. The lower the Pause time higher the mobility. The number of sources is 100 , with varying Pause time is used for this analysis. The Packet Delivery ratio is based on the number of packets delivered and number of packets sent. Higher the Packet Delivery Ratio(PDR) better is the performance of the Cluster. The Modified Weighted Clustering Algorithm(MWCA) technique approaches a ratio of one or $100 \%$ delivery as the network becomes more static. Only under dynamic conditions the packets collide or the packets are lost. As the pause time increases the Static nature is enhanced. For WCA and Lowest ID algorithm even as the nodes become static all the packets are not delivered.

The Graphs above show the better performance of Modified Weighted Clustering Algorithm in the case Throughput, more Stability and longer Life span [11]. Thus the Weighted Clustering Algorithm when modified and liner auto regression prediction is more efficient form of Clustering.

\section{CONCLUSION}

Effective utilization of power [6], Minimum wastage of Bandwidth, More Stable Clusters helps in improving the QOS in MANETS. Weighted Clustering Algorithm itself is improved with the use of mobility prediction in the Cluster Maintenance phase. 


\section{REFERENCES}

[1] Ali Bokar, Muslim Bozyigit, \& Cevat Sener (2009). Scalable Energy-Aware Dynamic Task Allocation. International Conference on Advanced Information Networking and Applications Workshops.

[2] Basagni .S. (1999). Distributed Clustering for Ad Hoc Networks. International Symposium on Parallel Architectures, Algorithms and Networks, pp. 310- 315.

[3] Dhurandher.S.K., \& Singh.G.V. (2006). Power Aware Clustering Technique in Wireless Ad Hoc Networks, International Symposium on Ad Hoc and Ubiquitous Computing, ISAUHC '06

[4] Dhurandher S.K., \& Singh .G.V. (2005). Weight Based Adaptive Clustering in Wireless Ad Hoc Networks. IEEE International Conference on Personal Wireless Communications, New Delhi, India, 95-100.

[5] Gerla .M., \& Tsai .J. T. C. (1995). Multi-cluster Mobile Multimedia Radio Network. ACM/Baltzer Wireless Networks Journal 95, vol. 1, pp. 255-265.

[6] Hussein, Abu Salem.A.H., \& Yousef .A.O. (2008). A flexible weighted clustering algorithm based on battery power for Mobile Ad hoc Networks, IEEE International Symposium on Industrial Electronics.

[7] Jieying Zhou, Jianfeng Chen, Weicong Xie, \& Jing Li (2007) Improved Weight Clustering Algorithm for IDs in Mobile AdHoc Network, Wireless Communications, Networking and Mobile Computing, 2007. WiCom '07, International Conference on volume , Issue , 21-25.

[8] Jing Wu , Guo-chang Gu, \& Guo-zhao Hou (2009). A Clustering Algorithm Considering on a Hierarchical Topology's Stability for Ad Hoc Networks. First International Workshop on Education Technology and Computer Science.

[9] Mohapatra.P., \& Krishnamurthy.S.V. (2005). Ad Hoc Networks Technologies and Protocols, Springer Science + Business Media.

[10] Ramanathan.R,. \& Redi.J. (2002). A Brief Overview of Ad Hoc Networks: Challenges and Directions, IEEE Communication Magazine, 40(5).

[11] Sharmila Anand John Francis, Elijah Blessing Rajsingh, \& Giss George (2009). Enhancing Stability of Network through Clustering in Mobile Ad hoc Networks. Third International Conference on Next Generation Mobile Applications, Services and Technologies

[12] Sucec.J., \& Marsic.I. (2002). Clustering overhead for hierarchical routing in mobile ad hoc networks, IEEE proceeding.

[13] Toh.c.k, \& Chai K Toh ( 2002). Ad Hoc Mobile Wireless Networks protocols and Systems, New Jersey:Prentice Hall PTR.

[14] Vieu .V.B., Nasser .N., \& Mikou .N. (2006). A Weighted Clustering Algorithm Using Local Cluster-heads Election for QoS in MANETs. IEEE GLOBECOM.

[15] Wang. .Y.,Chen .H., Yang X., \& Zhang .D. (2007). Wachm: Weight based adaptive clustering for large scale heterogeneous manet. Communications and Information Technologies, ISCIT '07. pp. 936-941.

[16] Yan Shuailing, Jiang Huawei, \& Wang Gaoping (2008). An Improved Clustering Algorithm Based on MANET Network. International Symposium on IT in Medicine and Education

[17] Yang .W.d., \& Zhang .G.z. (2007). A Weight-based Clustering Algorithm for mobile Ad Hoc network. Third International Conference on Wireless Communications.

[18] [Yoon-cheol Hwang, Yoon-Su Jeong, Sang-Ho Lee, Jeong-Young Song, \& Jin-Il Kim1 ( 2008). Advanced Efficiency and Stability Combined Weight Based Distributed Clustering Algorithm in MANET, Future Generation Communication and Networking.

[19] Yu .J.P. \& Chong P.H.J. (2005).A Survey of Clustering Schemes for Mobile Ad Hoc Networks. IEEE Communications Surveys and Tutorials, Vol. 7, No. 1, pp. 32-48.

[20] Yuji Kawai, \& Iwao Sasase (2008). A Stable Clustering Scheme by Prediction of the Staying Time in a Cluster for Mobile Ad Hoc Networks. APCC. 